\title{
Coordinated Tuning of the Parameters of PSS and POD Controllers Using Bioinspired Algorithms
}

\author{
Ednei Luiz Miotto ${ }^{(\mathbb{D}}$, Percival Bueno de Araujo ${ }^{(0)}$, Elenilson de Vargas Fortes ${ }^{(1)}$, \\ Bruno Rafael Gamino (1), and Luis Fabiano Barone Martins (i)
}

\begin{abstract}
This paper presents a technique based on the novel bat algorithm (NBA) for the design of the parameters of power system stabilizers and the generalized Unified power flow controllerpower oscillation damping in a multimachine power system. The objective is to ensure minimal damping rates to low-frequency electromechanical oscillation modes. Simulations were carried out on the New England system to compare the performance of the NBA with three other techniques: particle swarm optimization, bacterial foraging optimization oriented by particle swarm optimization, and the bat algorithm. For the cases analyzed in this paper, the NBA produced results superior to other techniques. Moreover, the proposed methodology was able to obtain solutions with high damping levels, which showed to be robust when a wide range of loading conditions of the power system were considered.
\end{abstract}

Index Terms-Novel bat algorithm (NBA), power system stabilizers (PSSs), power oscillation damping (POD), small-signal stability.

\section{INTRODUCTION}

$\mathbf{O}$ VER the years, small electric power systems (EPSs) have been interconnected to cover increasingly larger areas, thereby contributing to the economic development and the welfare of the general population. Compared to isolated systems, interconnected electrical systems are more robust and have less impact when rain shortages affect electric power generation. Despite this advantage, interconnections between systems also

Manuscript received October 19, 2017; revised January 5, 2018 and February 23, 2018; accepted March 26, 2018. Date of publication April 5, 2018; date of current version July 17, 2018. Paper 2017-IACC-1253.R2, presented at the 2016 12th IEEE International Conference on Industry Applications, Curitiba, Brazil, Nov. 20-23, and approved for publication in the IEEE TRANSACTIONS ON INDUSTRY APPLICATIONS by the Industrial Automation and Control Committee of the IEEE Industry Applications Society. This work was supported by the Brazilian Federal Agency for the Support and Evaluation of Graduate Education. (Corresponding author: Ednei Luiz Miotto.)

E. L. Miotto is with the Department of Electronic Engineering, Federal Technological University of Paraná Toledo, Paraná 85902-490, Brazil (e-mail: edneimiotto@utfpr.edu.br).

P. B. de Araujo is with the Department of Electrical Engineering, São Paulo State University, Ilha Solteira 15385-000, Brazil (e-mail: percival@dee. feis.unesp.br)

E. de Vargas Fortes is with the Goiás Federal Institute of Education, Science, and Technology, Jataí 75804-714, Brazil (e-mail: elenilson.fortes@ifg.edu.br).

B. R. Gamino is with the Department of Electrical Engineering, São Paulo State University, Ilha Solteira 15385-000, Brazil (e-mail: gaminobr@ gmail.com).

L. F. B. Martins is with the Paraná Federal Institute of Education, Science, and Technology, Jacarezinho 86400-000, Brazil (e-mail: luis.martins @ ifpr.edu.br).

Color versions of one or more of the figures in this paper are available online at http://ieeexplore.ieee.org.

Digital Object Identifier 10.1109/TIA.2018.2824249 have some drawbacks. The fact that several devices with different dynamics are integrated into the same electrical network makes the modeling and operation of the system more complex, which directly affects the capacity to maintain the stability of the electrical system. Kundur et al. [1] have defined stability as the ability of the system to remain in equilibrium under normal operating conditions or when subjected to perturbations. If the perturbation is small (e.g., load variations during the day leading to the need for power generation adjustments), it is termed small-signal stability, which is the focus of this paper.

A small perturbation is a small deviation in the state of the system that allows linearization of all the equations describing this system around a static equilibrium point. Thus, the electrical system can be represented by linear models. To model the EPS, this paper used the power sensitivity model, whose fundamental principle is based on the balance of active and reactive power at each bus during the entire dynamic process [2]. These small perturbations in the system may lead to the emergence of lowfrequency oscillatory modes that are classified according to their frequency; the most important modes are local $(0.8-2.0 \mathrm{~Hz})$ and interarea $(0.1-0.7 \mathrm{~Hz})$ modes [3]. The presence of these oscillatory modes may lead the EPS to instability.

To introduce the additional damping torque to the system, controllers called power system stabilizers (PSSs) are installed in control circuits of automatic voltage regulators (AVR), where stabilizing signals are inserted that act on the generating unit's voltage control output [4], [5]. Thus, these stabilizers are often used to increase the damping of the low-frequency oscillatory modes [6]-[9].

With a growing demand for electrical energy and increasingly stringent laws regarding environmental and social impacts, there are factors that motivate the most rational and optimized use of existing EPSs and contribute to the introduction of new automatic compensation devices such as flexible ac transmission systems (FACTS). The use of FACTS devices improves the control and operational safety of EPSs [10]. Studies have shown that the supplementary damping of EPS oscillations can be provided when a FACTS device is coupled to a power oscillation damping (POD) controller, especially with respect to interarea mode oscillations [11].

However, for these supplementary controllers to damp local and interarea oscillatory modes effectively, their parameters must be correctly tuned. Several techniques for adjusting these controllers using bioinspired optimization methods has gained prominence in recent years. In this case, the main goal is to tune 
the stabilizers in a coordinated way in order to maximize the system damping. In this context, genetic algorithms (GAs) were used in [12] to allocate and design the parameters of the PSSs and unified power flow controllers (UPFC) under different operating conditions; in [13] to tune the parameters of the PSS and IPFC-POD controllers using a Specialized Chu-Beasley's GA; and in [14] to optimize the PSSs by combining participation factors with GAs.

Bioinspired optimization methods based on social and cooperative behaviors found in nature such as bacterial foraging optimization (BFO) [15], [16], particle swarm optimization (PSO) [17] have also been used for the design of the parameters of PSS and POD associate with different FACTS devices. In [18], the artificial bee colony algorithm was used for the design of the PI-UPFC-POD and PSS damping controllers in a multimachine power system. A cuckoo search algorithm was proposed in [19] to design the PSSs considering various operational conditions and disturbances in the power system.

In order to take advantage of different techniques, hybrid methodologies have also been proposed. The hybrid algorithm bacteria foraging optimization and PSO algorithm were used in [20] to tune the PSSs and SSSC, and in [21] for the design of TCSC connected in electrical power systems.

Recently, the bat algorithm (BA) [22] has been tested to tune the PSS controller parameters, and its performance has been compared with both ant algorithms (ACO) and GA to mitigate low-frequency oscillatory modes in a multimachine power system. Although the BA has presented promising results, it has some shortcomings that need special attention as anticipated in [22]. The classic BA does not take diverse foraging habitats into account, which can arrest the algorithm in a local optimum. Moreover, the influence of the Doppler effect on the frequency of the signal that bats emit and receive might affect the local search process. These two problems have recently been overcome in a variation of the classic BA called the novel BA (NBA) [23].

In this context, to provide damping to unstable or low-damped modes of an EPS and, thereby, increasing its stability limits, this paper used the NBA-based algorithm to perform the design of the parameters of the supplementary damping controllers, PSS, and generalized unified power flow controller-power oscillation damping (GUPFC-POD). It is important to note that this paper is a continuation of an earlier study [16] that had its results put forward at an IEEE conference. In [16], Miotto et al. performed the simulations in a smaller power system and did not consider any variation of the test system load. In addition, they analyzed only three methods (PSO, BFO, and BF-PSO) and considered a lower desired damping index than the current one. Thus, the main contributions of this paper are as follows.

1) It implements computationally a NBA-based algorithm to perform the design of the PSS and GUPFC-POD controllers.

2) It validates the NBA-based algorithm as an optimization technique for adjusting the parameters of the PSS and GUPFC-POD controllers.

3) It analyzes the efficiency of the PSS and GUPFC-POD controllers when the goal is to insert damping to local and interarea oscillation modes.

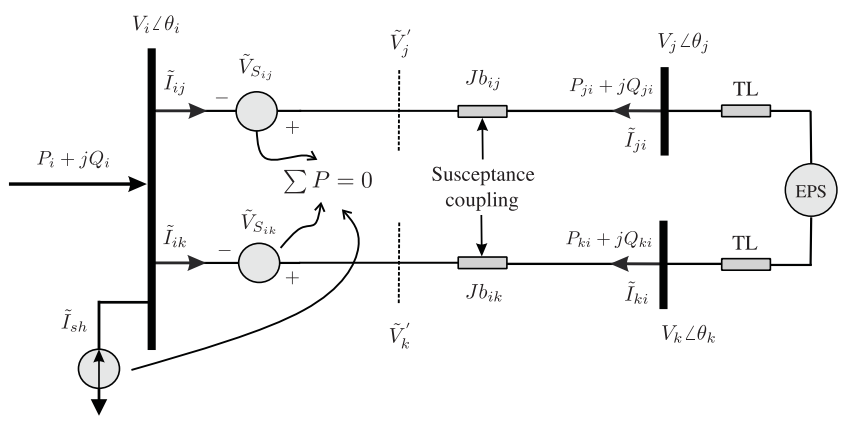

Fig. 1. Equivalent circuit of the GUPFC.

Besides that, this paper addresses different damping factors and system load conditions to evaluate the efficiency of the analyzed methods to maintain small-signal stability in a mediumsized system, known in the literature as New England.

The following sections comprise the rest of this paper: Section II models the GUPFC FACTS device. Section III presents the structure of the supplementary damping controller. Section IV provides an overview of the optimization problem. Section V describes the four tuning techniques for PSS and GUPFC-POD controllers, and Section VI details the results of the simulations and provides a statistical study comparing the previously presented techniques. Finally, Section VII discusses some conclusions.

\section{Generalized Unified Power Flow Controller}

This section presents the power injection model that represents the GUPFC in the power flow. This model is based on a configuration with three voltage source converters (VSCs) connected via a common dc link [24].

Fig. 1 represents the equivalent circuit of the GUPFC. This circuit is modeled by two things: 1 ) an ideal current source $\tilde{I}_{\mathrm{sh}}$ connected in shunt to the common bus $i$ of the installation; and 2) two series voltage sources that inject synchronous voltages with controllable amplitudes and angles $\tilde{V}_{S_{\text {in }}}$ into the system. In series with each source is susceptance $b_{\text {in }}(n=j, k)$, which models the coupling transformers of the VSC to the ac network. This configuration allows the management of four active and reactive power flows in both lines in which it is installed in addition to the voltage of the common installation bus. The GUPFC controls the power flow in the transmission lines (TLs), where it is installed by controlling the amplitude and angle of the injected series voltage $\tilde{V}_{S_{\text {in }}}$, where $n=j, k$.

The magnitudes $\tilde{V}_{j}^{\prime}$ and $\tilde{V}_{k}^{\prime}$ correspond to the voltages in fictitious buses used in the modeling. $P_{i}$ and $Q_{i}$ are the complex power injections into the bus $i$ of the system; $P_{j i}$ and $Q_{j i}$ are the flows of active and reactive power in the branch $i-j$ leaving bus $j ; P_{k i}$ and $Q_{k i}$ are the flows of active and reactive power in the branch $i-k$ leaving bus $k$; and $I_{j i}$ and $I_{k i}$ are the currents in branches $i-j$ and $i-k$ leaving buses $j$ and $k$, respectively.

$\tilde{V}_{S_{\text {in }}}$ can be decomposed into two components: one in quadrature $V_{p n}$ and the other in phase $V_{q n}$ with the voltage $\tilde{V}_{i}$ of the common installation bus, where $n=j, k$. Similarly, the ideal current shunt source $\tilde{I}_{\text {sh }}$ can be decomposed into two 
components, one in phase $i_{p}$ and the other in quadrature $i_{q}$ with the voltage $\tilde{V}_{i}$ of the common bus of installation $i$.

If losses are disregarded, all active power supplied by VSC1 shunt converter will be equal to the power delivered to the alternating current system by the series converters (VSC2 and VSC3). This constraint is presented in (1), which is known as the active power invariance of the GUPFC

$$
P_{\mathrm{sh}}=-\sum_{(m=i, j, k)} P_{s e, m}
$$

Considering the aforementioned constraint, the equivalent circuit of the GUPFC illustrated in Fig. 1, arrives in the power injection model for the GUPFC FACTS device [(2)-(5)], which represents the sum of the series contributions of the synchronous voltage sources and the shunt contribution of the ideal current source

$$
\begin{aligned}
P_{i}^{\mathrm{inj}} & =-\sum_{(n=j, k)} b_{\text {in }} V_{n}\left(V_{q n} \sin \left(\theta_{\text {in }}\right)+V_{p n} \cos \left(\theta_{\text {in }}\right)\right) \\
Q_{i}^{\mathrm{inj}} & =-V_{i} \sum_{(n=j, k)} b_{\text {in }} V_{q n}-V_{i} i_{q} \\
P_{n}^{\mathrm{inj}} & =b_{\text {in }} V_{n}\left(V_{q n} \sin \left(\theta_{\text {in }}\right)+V_{p n} \cos \left(\theta_{\text {in }}\right)\right) \\
Q_{n}^{\mathrm{inj}} & =b_{\text {in }} V_{n}\left(V_{q n} \cos \left(\theta_{\text {in }}\right)-V_{p n} \sin \left(\theta_{\text {in }}\right)\right)
\end{aligned}
$$

where $P_{i}^{\mathrm{inj}}$ and $Q_{i}^{\mathrm{inj}}$ are the active and reactive power injections in the common bus of the installation. $P_{n}^{\mathrm{inj}}$ and $Q_{n}^{\mathrm{inj}}$ (with $n=j, k$ ) are injections of active and reactive power in buses $j$ and $k$ of the installation, respectively. Further details on the power injection model can be seen in [16]. Section II-A presents the dynamic control structure of the GUPFC.

\section{A. Structure of the GUPFC Control System}

Fig. 2 shows the control structure used to represent the dynamics of the GUPFC device, where each PI controller is related to a variable of the voltage sources that represent the series converters $\left(V_{p j}, V_{q j}, V_{p k}\right.$, and $\left.V_{q k}\right)$ and the shunt converter $I_{q}$ [13], [16].

In Fig. 2, the gains $K_{1}^{p i}$ to $K_{4}^{p i}$ and the time constants $T_{1}^{p i}$ to $T_{4}^{p i}$ are the parameters of the PI controllers. The gain $K_{r}$ and the time constant $T_{r}$ are related to the regulation of the voltage in the common bus of the GUPFC installation. The time constant $T_{\text {gupfc }}$ is related to the processing time of the device control system, whose range varies from 1 to $10 \mathrm{~ms}$ [10]. The supplementary control signal $V_{\text {sup }}^{2}$ (of the POD controller) modulates the quadrature component $V_{p j}$ of VSC2 and is intended to provide additional damping to oscillations of the EPS.

$P_{n}^{\text {ref }}$ and $Q_{n}^{\text {ref }}$ are the values specified by the system operator for the active and reactive power flows in TLs $i-n ; P_{n}^{c}$ and $Q_{n}^{c}$ are the active and reactive power flows controlled in the two TLs that the GUPFC regulates, where $n=j, k . V_{i}^{\text {ref }}$ is the reference voltage on the common bus of the FACTS installation and $V_{i}$ is the voltage in bus $i$ controlled by modulating the current $I_{q}$ in quadrature of the VSC1 converter.
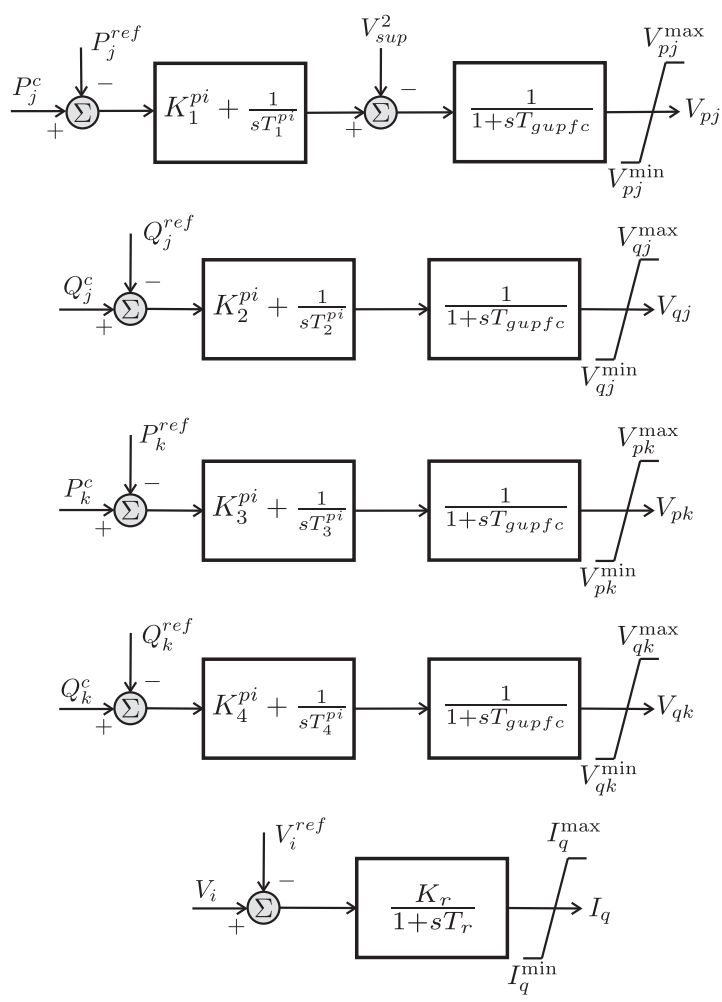

Fig. 2. GUPFC control system structure.

Fig. 2 describes the set of differential equations represented in (6)-(14) that define the dynamic behavior of the GUPFC device

$\dot{V}_{p j}=\frac{K_{1}^{p i}}{T_{\text {gupfc }}}\left(P_{j}^{\text {ref }}-P_{j}^{c}\right)+\frac{X_{1}}{T_{\text {gupfc }}}-\frac{V_{\text {sup }}^{2}}{T_{\text {gupfc }}}-\frac{V_{p j}}{T_{\text {gupfc }}}$

$\dot{X}_{1}=\frac{1}{T_{1}^{p i}}\left(P_{j}^{\mathrm{ref}}-P_{j}^{c}\right)$

$\dot{V}_{q j}=\frac{K_{2}^{p i}}{T_{\text {gupfc }}}\left(Q_{j}^{\text {ref }}-Q_{j}^{c}\right)+\frac{X_{2}}{T_{\text {gupfc }}}-\frac{V_{q j}}{T_{\text {gupfc }}}$

$\dot{X}_{2}=\frac{1}{T_{2}^{p i}}\left(Q_{j}^{\mathrm{ref}}-Q_{j}^{c}\right)$

$\dot{V}_{p k}=\frac{K_{3}^{p i}}{T_{\text {gupfc }}}\left(P_{k}^{\text {ref }}-P_{k}^{c}\right)+\frac{X_{3}}{T_{\text {gupfc }}}-\frac{V_{p k}}{T_{\text {gupfc }}}$

$\dot{X}_{3}=\frac{1}{T_{3}^{p i}}\left(P_{j}^{\mathrm{ref}}-P_{j}^{c}\right)$

$\dot{V}_{q k}=\frac{K_{4}^{p i}}{T_{\text {gupfc }}}\left(Q_{k}^{\mathrm{ref}}-Q_{k}^{c}\right)+\frac{X_{4}}{T_{\text {gupfc }}}-\frac{V_{q k}}{T_{\text {gupfc }}}$

$\dot{X}_{4}=\frac{1}{T_{4}^{p i}}\left(Q_{k}^{\mathrm{ref}}-Q_{k}^{c}\right)$

$\dot{I}_{q}=\frac{K_{r}}{T_{r}}\left(V_{i}^{\mathrm{ref}}-V_{i}\right)-\frac{I_{q}}{T_{r}}=\frac{1}{T_{r}}\left(K_{r}\left(V_{i}^{\mathrm{ref}}-V_{i}\right)-I_{q}\right)$. 


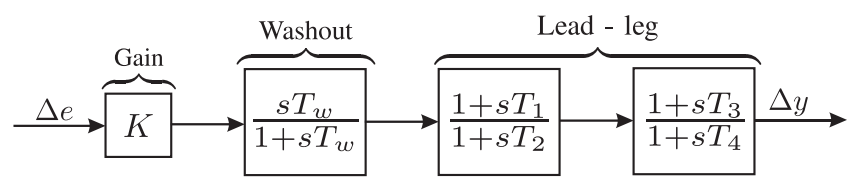

Fig. 3. Configuration of the PSS and POD controllers.

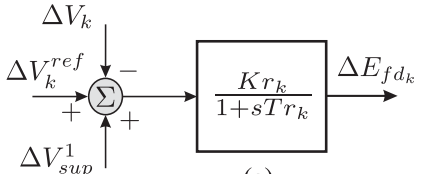

(a)

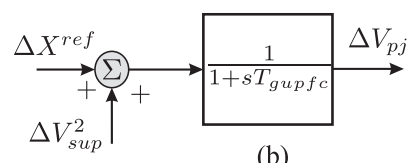

(b)
Fig. 4. Dynamic model of the PSS and POD. (a) Control loop of the AVRs. (b) Quadrature voltage component of the secondary converter of the GUPFC.

\section{SUPPLEMENTARY DAMPING CONTROLLERS PSS AND POD}

In order to introduce damping of the oscillations of the EPS, it is necessary to include the supplementary damping controllers such as the PSS and the POD. Fig. 3 shows the generic structure that can be used for the two controllers.

In Fig. 3, $\Delta e$ and $\Delta y$ are the input and output signals, respectively. The gain $K$ acts on the amplification or attenuation of the processed signal. The washout filter, formed by the time constant $T_{w}$, has the function of attenuating the variation of the input signal and zeroing the controller response in a steady state. The lead-lag compensator blocks are responsible for phase compensation of the oscillatory mode of interest using the time constants $T_{1}, T_{2}, T_{3}$, and $T_{4}$.

For the $\Delta e$ input signal, the PSS uses variations in the angular velocity $\Delta \omega_{k}$ of generator $k$. The POD input signal is the deviation of active power flow $\Delta P_{k m}$ in the TL adjacent to the installation of the GUPFC FACTS device. The PSS output signal $\Delta y$ is the voltage $\Delta V_{\text {sup }}^{1}$ and is introduced as an additional signal to the control loop of the AVR represented in this paper using a first-order model [see Fig. 4(a)]. The POD generates a stabilizing signal $\left(\Delta V_{\text {sup }}^{2}\right)$, which is introduced in the control loop of the GUPFC to modulate the component in quadrature $V_{p j}$ of the secondary converter of the device [see Fig. 4(b)], as presented in [13].

\section{OVERVIEW OF THE OPTIMIZATION PROBLEM}

This paper considers a multimachine EPS with $n$ PSS controllers and a GUPFC FACTS device equipped with a POD. In the computational model, each individual represents a possible solution to the problem, which is coded as a vector (see Fig. 5) composed of the gains and time constants of the controllers.

Note that the first $n$ positions of the individual shown in Fig. 5 are destined to the $n$ constants of time $T_{1 n}-T_{2 n}$ of the $n$ PSS controllers installed in $n$ synchronous machines. Position $n+1$ refers to the $n K_{\text {pss }}$ gains $n$ of the PSS controllers. Continuing this sequence, the last positions of the vector are reserved for the time constant $T_{p 1}-T_{p 2}$ and for the $K_{\text {pod }}$ gain of the POD controller, respectively. It is usual to adopt $T_{1 n}\left(T_{p 1}\right)=T_{3 n}$ $\left(T_{p 3}\right)$ and $T_{2 n}\left(T_{p 2}\right)=T_{4 n}\left(T_{p 4}\right)$ [25].

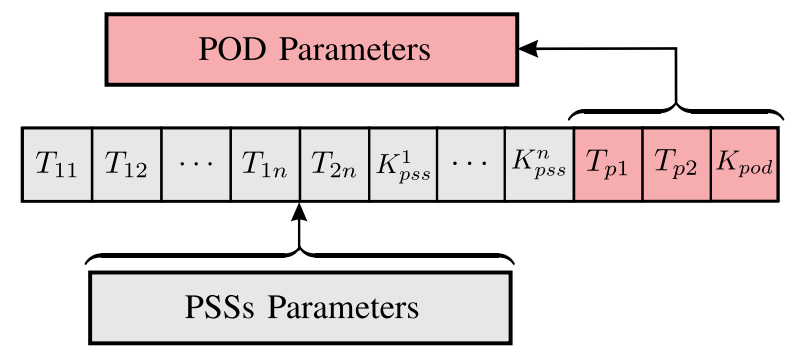

Fig. 5. Representation of an individual in the NBA.

The objective of this problem is to minimize (15) the subject to the set of constraints defined in (16)-(19). The objective function (15) used in this paper consists of minimizing the module of the difference between the calculated damping $\xi_{i}^{\text {cal }}$ and the desired damping $\xi^{\text {des }}$, where $n p$ is the number of eigenvalues of interest (local mode and interarea mode). The constraints (16) and (17) define the maximum and minimum limits of the time constants and gains of the PSS and POD supplementary controllers. Finally, the constraint (18) defines a minimum damping $\left(\xi_{i}^{\mathrm{min}}\right)$ for eigenvalues of interest greater than or equal to the desired damping $\left(\xi^{\text {des }}\right)$ specified by the design, while the constraint (19) determines a minimum value for the real part of the eigenvalues of interest

$$
\begin{aligned}
& F_{O}=\min \sum_{i=1}^{n p}\left|\xi_{i}^{\mathrm{cal}}-\xi^{\mathrm{des}}\right| \\
& T_{1 n}^{\min } \leq T_{1 n} \leq T_{1 n}^{\max } ; T_{2 n}^{\min } \leq T_{2 n} \leq T_{2 n}^{\max } ; \\
& K_{\mathrm{pss}}^{\min } \leq K_{\mathrm{pss}} \leq K_{\mathrm{pss}}^{\max } \\
& T_{p 1}^{\min } \leq T_{p 1} \leq T_{p 1}^{\max } ; T_{p 2}^{\min } \leq T_{p 2} \leq T_{p 2}^{\max } ; \\
& K_{\mathrm{pod}}^{\min } \leq K_{\mathrm{pod}} \leq K_{\mathrm{pod}}^{\max } \\
& \xi_{i}^{\min } \geq \xi^{\text {des }} \\
& \sigma_{i} \leq \sigma^{\mathrm{min}} .
\end{aligned}
$$

\section{TeChNique FOR THE DESIGN OF the PSS AND POD CONTROLLERS}

This section outlines the approaches of four bioinspired techniques to tune the parameters of the PSS and GUPFC-POD controllers. This paper gives a special highlight to the NBA approach.

\section{A. Particle Swarm Optimization}

The first of the four techniques used is PSO, which was initially developed in [26]. It is based on the metaphor of social behavior that was obtained from observations of groups of animals in nature such as flocks of birds and shoals of fish. These populations of individuals, more commonly called particles, present limited individual capacity but intelligent collective behavior.

At each iteration of the algorithm, the particles (possible solutions to the problem) move through a search space ( $n$-dimensional region) influenced by their best previous 
experiences $\left(p_{\text {Lbest }}^{t}\right)$ and the best experience of neighboring particles $\left(p_{\text {Global }}^{t}\right)$ in the search for the optimal point.

In order to direct the flight of the particles to more promising regions in the search space, the algorithm uses a $\delta$ inertia coefficient to increase the search capabilities. $\delta$ values ranging between 0.8 and 1.20 give the PSO technique better chances of finding the best overall solution with a moderate number of iterations. Each particle has a position represented by position vector $x_{i}^{t}$ (where $i$ is the index of the particle) and a speed represented by speed vector $v_{i}^{t}$. Equation (20) updates the speed during interaction $t$ from the previous speed

$$
v_{i}^{t+1}=\delta v_{i}^{t}+\rho_{1} r_{1}^{t}\left(p_{\text {Lbest }}^{t}-x_{i}^{t}\right)+\rho_{2} r_{2}^{t}\left(p_{\text {Global }}^{t}-x_{i}^{t}\right) .
$$

Equation (21) calculates the new position from the previous position and the current speed

$$
x_{i}^{t+1}=x_{i}^{t}+v_{i}^{t+1} .
$$

The acceleration coefficients $\rho_{1}$ and $\rho_{2}$ along with random values $r_{1}$ and $r_{2}$ exert the stochastic influence of cognitive and social factors on the particle's velocity, respectively. According to [27], when $\rho_{1}+\rho_{2}<4.0$, a swarm of particles can lead to slow convergence or even nonconvergence. However, if $\rho_{1}+\rho_{2}>4.0$, the convergence of the method will be rapid and guaranteed. This paper considered $\rho_{1}+\rho_{2}=4.0$, where $\rho_{1}=\rho_{2}=2.0$. The pseudocode of the PSO algorithm can be found in [16].

\section{B. BFO Oriented by PSO}

The BF-PSO algorithm is the second technique this paper evaluated. The BF-PSO is a hybrid algorithm that combines the advantages of two techniques: The PSO shown in the previous section and the BFO presented in this section. That is, the ability of the PSO to exchange social information is pooled with the BFO decision making ability to find new solutions (e.g., the richest food location ) through changes in the elimination and dispersal step [28].

This section presents a brief summary of the BFO algorithm. Four processes explain the foraging strategy of Escherichia coli bacteria: 1) chemotaxis; 2) swarming; 3 ) reproduction; and 4) elimination dispersal [15]. Each bacterium can move in two ways, swimming or tumbling. This is called chemotaxis. By swimming, the bacteria try to converge in a particular region. By tumbling, the bacteria move randomly around the region. Equation (22) shows the displacement of a bacterium in a specific displacement step $D(i)$ and in a random direction specified by a given tumble $\oslash(j)$

$$
\Theta^{i}(j+1, k, l)=\Theta^{i}(j, k, l)+D(i) \oslash(j) .
$$

In (22), $\Theta^{i}(j, k, l)$ refers to the bacterium in the $i$ th position, the $j$ th chemotaxis step, the $k$ th stage of reproduction, and $l$ th stage of elimination and dispersal. The tumble process is shown in (23). It is composed of a random vector $\Delta(i)$ in which random values between -1 and 1 for each specific element

$$
\oslash(j)=\frac{\Delta(i)}{\sqrt{\Delta(i) \cdot \Delta^{T}(i)}} .
$$

This type of bacterium moves in an organized and structured manner, easily adapting to search regions. Equation (24) calculates the bacterium health at each displacement $\Theta^{i}(j, k, l)$, which represents the cost function of the optimization problem, including the portion $J_{c c}$ from the agglomeration step

$$
J(i, j+1, k, l)=J(i, j, k, l)+J_{c c}\left(\Theta^{i}(j+1, k, l)\right) .
$$

The second step, called agglomeration, is the tendency of the bacteria to organize and converge toward the same spot. The mathematical function for agglomeration, considering cell-cell signaling by attraction and repulsion, is defined as

$$
\begin{aligned}
& J_{c c}\left(\Theta^{i}(j+1, k, l)\right)=\sum_{i=1}^{S}-d_{\text {attract }} \\
& \quad \times \exp \left(-w_{\text {attract }} \sum_{i=1}^{d}\left(\Theta_{m}-\Theta_{m}^{i}\right)^{2}\right) \\
& +\sum_{i=1}^{S} h_{\text {repellant }} \times \exp \left(-w_{\text {repellant }} \sum_{i=1}^{d}\left(\Theta_{m}-\Theta_{m}^{i}\right)^{2}\right) .
\end{aligned}
$$

$J_{c c}\left(\Theta^{i}(j+1, k, l)\right)$ is a term added to the original cost function to represent a variant cost function over time, where $S$ is the number of bacteria, $d$ is the number of parameters to be optimized, and the coefficients $d_{\text {attract }}, h_{\text {repellant }}, w_{\text {attract }}, w_{\text {repellant }}$ are constants that govern the form of attraction or repulsion between bacteria. The value of the agglomeration function $J_{c c}$ tends to zero as long as there is a tendency for the bacteria to converge to a point of the best solution. This paper used the constant values found in [16], where $d_{\text {attract }}=h_{\text {repellant }}=0.1, w_{\text {attract }}=0.2$, and $w_{\text {repellant }}=0.3$.

The third stage is reproduction. This step occurs at the end of the chemotaxis process where bacteria are divided into two groups of equal size: the healthiest and least healthy bacteria. The healthiest bacteria reproduce and replace the least healthy bacteria, thereby maintaining a constant population. The health of bacteria is calculated by

$$
J_{\text {health }}^{i}=\sum_{j=1}^{N c} J(i, j, k, l) .
$$

A new stage of chemotaxis starts for each reproduction step and after this, a percentage of less healthy bacteria is eliminated. The fourth stage, called the elimination and dispersal stage, is a process of elimination of less healthy bacteria. Chemotaxis and reproduction begin again after elimination of the least healthy bacteria. More details about the BFO algorithm and its respective equations can be found in [15]. Details about the pseudocode of the BF-PSO algorithm can be found in [16].

\section{Bat Algorithm}

The BA is based on the advanced ability of echolocation of several bat species. Bats use echolocation to locate prey and to avoid obstacles, even under conditions of total darkness, by emitting sound signals with variable loudness and frequencies. 
The pulse rate and the loudness of the sounds a bat emits vary with its hunting strategy. When a bat locates a possible prey, the pulse rate $r_{i}$ and loudness $A_{i}$ of the sound signal increase to avoid the loss of the prey. After catching its prey, the bat's amplitude decreases.

In the classic BA, the movement of the virtual bat through the search space is given by following equations [29]:

$$
\begin{aligned}
f_{i} & =f_{\min }+\left(f_{\max }-f_{\min }\right) \beta \\
v_{i}^{t+1} & =v_{i}^{t}+\left(x_{i}^{t}-x_{*}\right) f_{i} \\
x_{i}^{t+1} & =x_{i}^{t}+v_{i}^{t+1} .
\end{aligned}
$$

In (27)-(29), $v_{i}^{t+1}$ and $x_{i}^{t+1}$ are, respectively, the speed and position of the $i$ th bat in iteration $t ; f_{i}$ is the variation in the frequency of the $i$ th bat; the upper and lower limits of the $i$ th bat are $f_{\max }$ and $f_{\min }$, respectively, which depends on the domain size of the problem of interest; $\beta \in[0,1]$ is a random number drawn from a uniform distribution; and $x_{*}$ is the best solution found in iteration $t$.

Equation (30) illustrates how the local search process can generate new temporary solutions around the current best solution. The pulse rate controls this process, and the loudness controls the acceptance of these new solutions

$$
x_{i}^{t+1}=x_{*}+\zeta A_{\text {meam }}^{t}
$$

where $\zeta$ is a random number extracted from a uniform distribution belonging to the set $[-1,1]$ and $A_{\text {meam }}^{t}$ is the average of the volume of all bats at time $t$. If during the local search process, the volume $A_{i}>$ rand $(0,1)$ and $F\left(x_{i}\right)<F(x)$, then the volume $A_{i}$ and the pulse emission rate $r_{i}$ are updated according to (31) and (32), and the objective function is also updated

$$
\begin{aligned}
& A_{i}^{t+1}=\mu A_{i}^{t} \\
& r_{i}^{t+1}=r_{i}^{0}\left(1-e^{-\gamma t}\right) .
\end{aligned}
$$

The constants $\mu$ and $\gamma$ influence the amplitude of the sound signal and the pulse rate, respectively. Moreover, the adjustment of these two parameters affects the BA convergence rate. Finally, the best overall solution is updated and the process continues until the stop criterion is reached. The BA pseudocode, adapted to the problem of adjusting the parameters of the supplementary damping controllers, can be seen in [22].

\section{Novel BA}

The classic BA does not take into account the different foraging habitats and the influence of the Doppler effect. Thus, it restricts the virtual bat to a limited search space. The concepts of classical mechanics define the bat's trajectory, which results in a search for food in a single habitat and facilitates the entrapment of the bat into a local optimal point. The NBA solves these shortcomings.

Equation (33) incorporates the Doppler effect into the NBA, and each bat adaptively compensates according to the proximity of its target, which greatly improves the performance of the algorithm

$$
f_{r}=\frac{v \pm v_{r}}{v \pm v_{s}} f_{s}
$$

where $f_{s}$ and $f_{r}$ are the frequencies of the emitted and received signals, respectively, and $v_{r}, v_{s}$, and $v$ are the velocities of the receiver (bat), the source (target), and the wave speed in the medium, respectively. Supposing that the source and receiver are in motion, in (33) employs the positive $(+)$ sign when bats are approaching one another; otherwise, it uses the negative $\operatorname{sign}(-)$.

Another important modification in the NBA is the diversification of foraging habitats by the addition of quantum behavior in addition to classic BA's mechanical behavior. Based upon the theoretical aspects of quantum physics, it is possible to state that a particle with quantum behavior can appear in any position within the search space at a certain probability [30]. Thus, by introducing quantum theory into the algorithm, bats in the NBA can fetch food in a larger number of habitats. Meanwhile, the bats in classic BA can forage in a single habitat only.

1) Habitat selection: Habitat selection depends on several random phenomena. Thus, a stochastic decision will model habitat selection in order to facilitate understanding. If $P \in[0,1]$ represents the selection threshold and $R$, a random number of uniform distribution between $[0,1]$ is less than $P$, then bats will choose quantum behavior over a larger range of habitats for food searches. Otherwise, they will choose mechanical behavior with limited habitats.

2) Quantum behavior and mechanical behavior: Virtual bats in the NBA can search for food in a larger range of habitats. Their position during quantum behavior is formulated as follows:

$$
\begin{aligned}
& x_{i j}^{t+1}= \\
& \left\{\begin{array}{l}
g_{j}^{t}+\Phi \mid M \text { best }_{j}^{t}-x_{i j}^{t} \mid \ln \left(\frac{1}{u_{i j}}\right), \text { if } \text { rand }_{j}(0,1)<0.5 \\
g_{j}^{t}-\Phi \mid M \text { best }_{j}^{t}-x_{i j}^{t} \mid \ln \left(\frac{1}{u_{i j}}\right), \text { otherwise }
\end{array}\right.
\end{aligned}
$$

where $g_{j}^{t}$ is the best overall position of the bat population, $\Phi$ is an attraction-expansion coefficient, $u_{i j}$ is a nonzero number uniformly distributed between 0 and 1 , and $M$ best $_{j}^{t}$ is known as the mean best position, which is defined by the average of the $x_{i j}^{t}$ positions of all bats. Further details on the mathematical formulation of quantum behavior can be found in [30].

However, if the mechanical behavior is selected, there are some subtle changes in calculating the movement of the bats compared to the classic BA, as described by (35)-(38). An inertia weight $w$ is added to update the velocity and control the influence of the individual's previous velocity. Moreover, a compensation rate $C$, which varies with each individual, is added to the NBA so that bats can self-compensate the Doppler effect in echoes during the frequency calculation

$$
\begin{aligned}
& f_{i, j}=f_{\min }+\left(f_{\max }-f_{\min }\right) \operatorname{rand}(0,1) \\
& f_{i, j}^{*}=\frac{\left(c+v_{i, j}^{t}\right)}{c+v_{g, j}^{t}} f_{i, j}\left(1+C_{i} \frac{\left(g_{j}^{t}-x_{i, j}^{t}\right)}{\left|g_{j}^{t}-x_{i, j}^{t}\right|+\epsilon}\right)
\end{aligned}
$$




$$
\begin{aligned}
& v_{i, j}^{t+1}=w v_{i, j}^{t}+\left(g_{j}^{t}-x_{i, j}^{t}\right) f_{i, j}^{*} \\
& x_{i, j}^{t+1}=x_{i, j}^{t}+v_{i, j}^{t+1}
\end{aligned}
$$

where $w \in[0,1]$ is a uniform random vector, $\epsilon$ is a small computational constant to avoid division error by zero, and $C$ is a positive number such that $C \in[0,1]$. If $C$ is equal to zero, there is no compensation of the Doppler effect; if $C$ is equal to 1 , there is total compensation. The $C$ of each individual is randomly assigned. The speed of sound in air is $c(c=340 \mathrm{~m} / \mathrm{s})$, and $v_{g, j}^{t}$ is the velocity corresponding to the best overall position.

3) Local search: Different aspects are taken into account during the local exploration phase (e.g., noises generated by the bats themselves and by other things in the environment). In [23], a simplification of these aspects is performed. The relative loudness between the loudness of a specific bat and the average loudness of all bats is regarded as an influencing factor during the local exploration phase. Thus, the new position for each bat is generated as follows:

$$
\begin{gathered}
x_{i, j}^{t+1}=g_{j}^{t}\left(1+\operatorname{rand} n\left(0, \sigma^{2}\right)\right) \\
\sigma^{2}=\left|A_{i}^{t}-A_{\text {mean }}^{t}\right|+\epsilon .
\end{gathered}
$$

In (39)-(40), rand $n\left(0, \sigma^{2}\right)$ is a Gaussian distribution with mean zero and standard deviation $\sigma^{2}$, while $\epsilon$ is used to ensure that $\sigma^{2}>0$. The volume update and pulse emission rate are the same as in the classic BA. In addition, when no bat finds a solution (i.e., prey) that is better than the previous solution during several intervals of time $(G)$, which means the bats are trapped in a local optimum, the proposed method forces the swarm to look for food elsewhere (i.e., explore new habitats). For this purpose, the volumes $A_{i}^{t+1}$ of all bats are initialized again and the pulse rate $r_{i}^{t+1}$ can be temporarily set to a high value, encouraging a global search.

As discussed in the previous sections, the NBA has five extra parameters compared to the classic BA: $P, G, C, \Phi$, and $w$, and their values are based on [23]. Algorithm 1 shows the NBA pseudocode.

\section{Simulations AND Results}

Simulations were performed in a test system known in the literature as New England to validate the tuning of the PSS and GUPFC-POD controllers found by the four techniques analyzed in this paper. The single-line diagram of the New England test system is shown in Fig. 6. This system consists of 10 generators, 41 buses (including two fictitious buses, $F_{1}$ and $F_{2}$, related with the FACTS device), and 48 TLs arranged in two areas: the equivalent generator 10 (New York system) compactly represents area 1, and the other generators (New England system) represent area 2. The full description of this system can be found in [31].

An Intel Core $i 72.93 \mathrm{GHz}$ computer with $8 \mathrm{~GB}$ RAM and Windows 7 64-bits operating system performed the simulations. The solution methods and the modeling presented in the previous sections were implemented in MATLAB, without the aid of any toolbox.

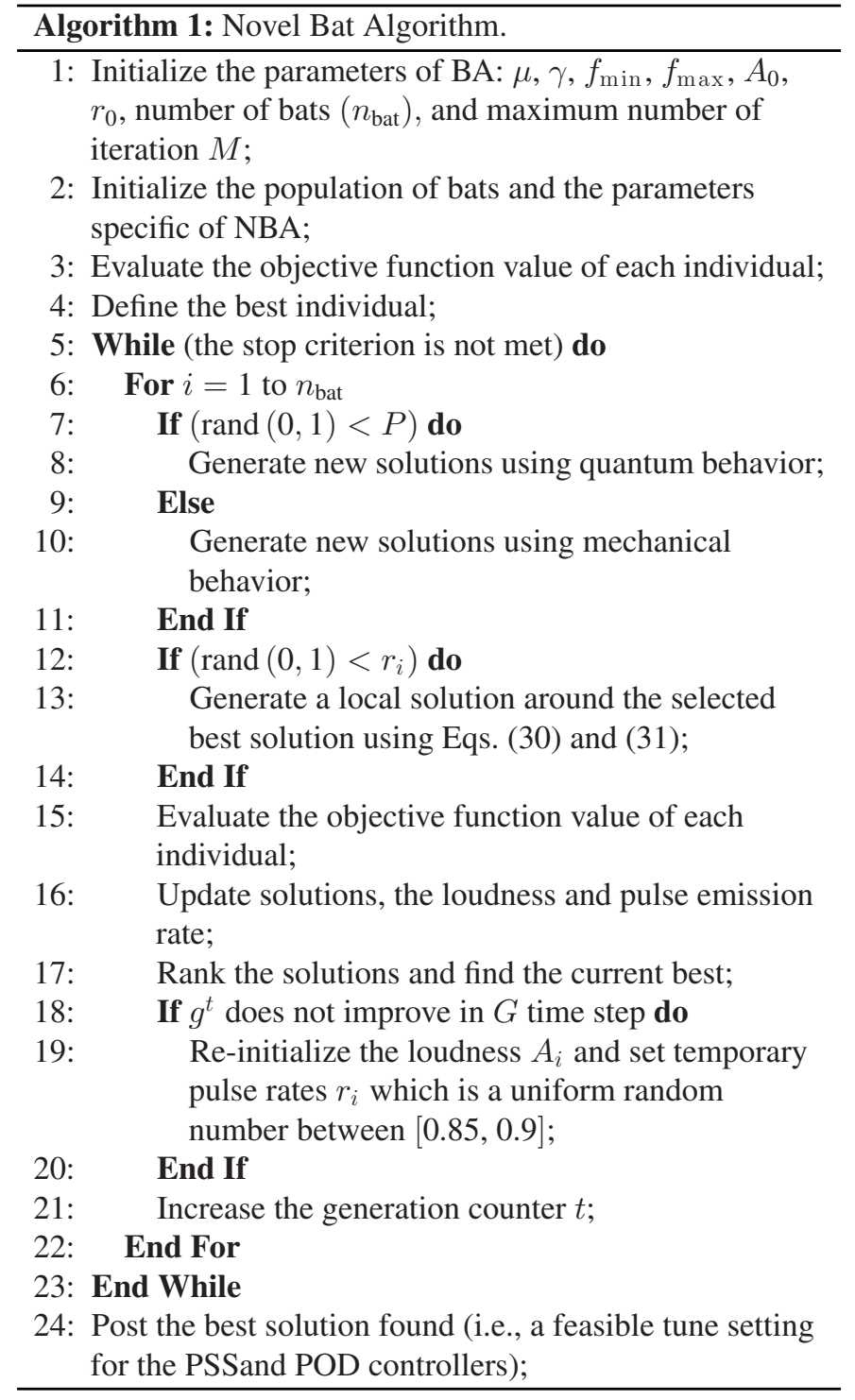

\section{A. Steady-State Analysis of the Power System}

As shown in Fig. 6, the researchers installed the GUPFC between buses $37-34$ and $37-36$, and selected bus 37 as the common bus. They selected bus 37 because of its technical and operational aspects, as the installation region contains most of the buses with a level of voltage below 0.95 p.u. (buses 12, $15,33,34,36$, and 37), which are highlighted by red circles (see Fig. 7). This location was also selected because it provides greater controllability and observability of the oscillatory mode of interest (interarea mode) than do the other locations.

The researchers defined Case I as the situation wherein the GUPFC does not exert control over the system; that is, it is turned OFF. Its variables have the following values (in p.u.): $V_{p j}=-2.68 \times 10^{-8}, V_{p k}=-2.68 \times 10^{-8}, V_{q k}=$ $-1.07 \times 10^{-6}, V_{q j}=-1.07 \times 10^{-6}$, and $I_{q}=-5.96 \times 10^{-5}$. These values are very close to zero, for the control variables of the series and shunt converters reinforce the FACTS lack of control over the active and reactive power flows in the test system. 


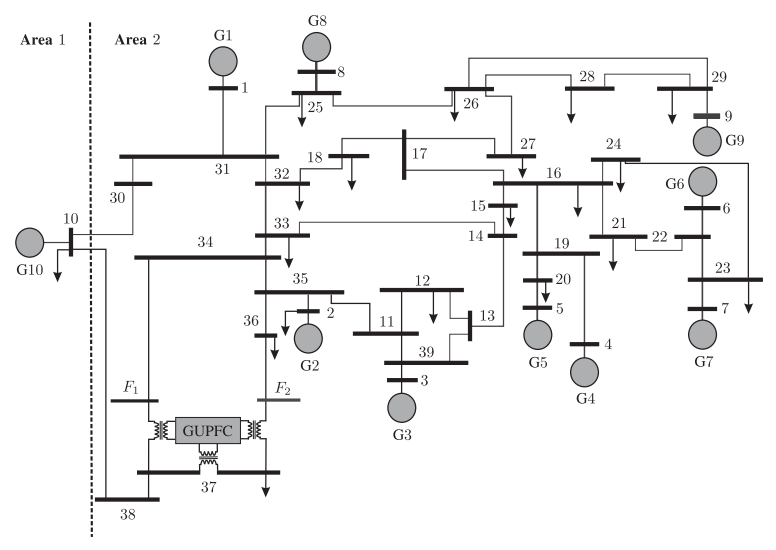

Fig. 6. One-line diagram of the New England system.

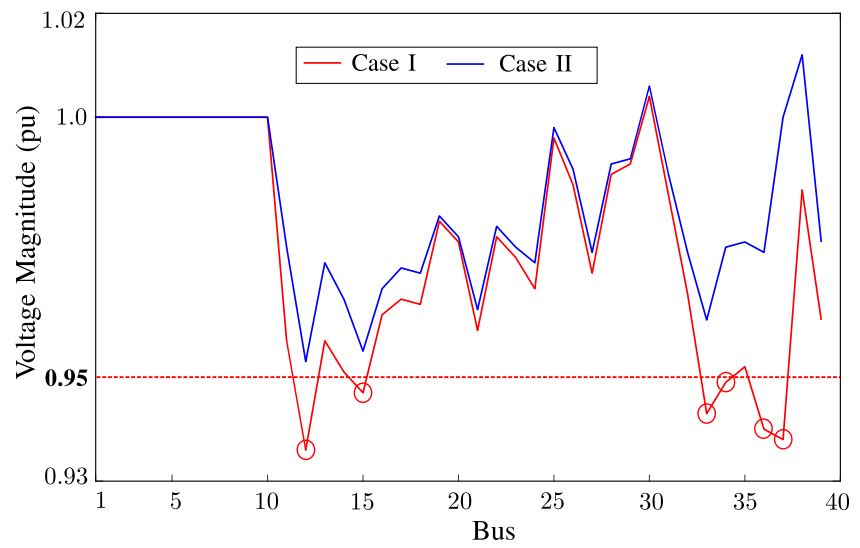

Fig. 7. Voltage profile before and after the installation of the GUPFC in the New England system.

To improve the voltage profile of the New England system, the GUPFC should act on the power flows (Case II) in the two TLs it controls $\left(F_{1}-34\right.$ and $\left.F_{2}-36\right)$ by providing a $10 \%$ increase in the active power flows in the two TLs controlled by the device, with the new values being -308.39 MW (Case I: $-280.36 \mathrm{MW}$ ) and $-228.97 \mathrm{MW}$ (Case I: $-208.16 \mathrm{MW}$ ), respectively. Meanwhile, the reactive flow in the $F_{1}-34$ stretches increased twofold, 83.01 MVar (Case I: $-41.5 \mathrm{MVar}$ ). In the $F_{2}$-36 stretch, the reactive flow increased ten times, $92.32 \mathrm{MVar}$ (Case I: -9.23 MVar). In both stretches, the reactive power flows were inverted in order to raise the voltage of the deficient buses. In addition, to maintain the voltage in bus 37 at 1.0 p.u., the GUPFC injected 273 MVAr into the common installation bus via its shunt converter.

After the action of the GUPFC (Case II), the researchers observed an overall improvement in the voltage profile of the New England system, with the voltages of all the buses within a safe range ( $\pm 5 \%$ of the nominal value) as can be seen in Fig. 7 . In this case, the control variables of the device assume the following values (in p.u.): $V_{p j}=-0.08237, V_{p k}=-0.0815$, $V_{q k}=-0.0163, V_{q j}=-0.01263$, and $I_{q}=2.727$.

The active power flows in the test system without the interference of the GUPFC and after its insertion can be seen in Fig. 8, which assists in the conclusion about the correct control of the active power flows and their invariance as presented in
TABLE I

DOMINANT EIGENVALUES, DAMPING COEFFiCIENTS $(\xi)$, AND NATURAL UNDAMPED FREQUENCIES $\left(\omega_{n}\right)$-CASE II

\begin{tabular}{lrrr}
\hline Modes & \multicolumn{1}{c}{ Eigenvalues } & $\xi$ (p.u.) & $\omega_{n}(\mathrm{~Hz})$ \\
\hline$L_{1}$ & $-0.241 \pm j 8.323$ & 0.0289 & 1.3251 \\
$L_{2}$ & $-0.189 \pm j 8.277$ & 0.0227 & 1.3177 \\
$L_{3}$ & $-0.266 \pm j 8.096$ & 0.0328 & 1.2892 \\
$L_{4}$ & $-0.232 \pm j 7.199$ & 0.0322 & 1.1464 \\
$L_{5}$ & $0.073 \pm j 6.839$ & -0.0107 & 1.0885 \\
$L_{6}$ & $0.180 \pm j 5.914$ & -0.0305 & 0.9417 \\
$L_{7}$ & $0.161 \pm j 6.395$ & -0.0252 & 1.0180 \\
$L_{8}$ & $-0.137 \pm j 6.484$ & 0.0211 & 1.0322 \\
$I_{9}$ & $-0.005 \pm j 3.560$ & 0.0013 & 0.5667 \\
\hline
\end{tabular}

(1). Moreover, the increase in the active power flow in the two TLs controlled by the GUPFC occurs because of the transfer of power between the two series converters.

\section{B. Dynamic Analysis of the Power System}

Using the Case II to define the current operational situation, the eigenvalues of the state matrix were calculated. Table I shows the oscillatory modes of interest $\left(\lambda_{i}=\sigma_{i} \pm j \omega_{i}\right)$, the damping coefficient $\left(\xi_{i}=-\frac{\sigma}{\left|\lambda_{i}\right|}\right)$, and the natural undamped frequency $\left(\omega_{n i}=\frac{\left|\lambda_{i}\right|}{2 \pi}\right)$. Note that the first eight are local modes and the last one is the interarea mode. A quick analysis of this table shows that although the GUPFC has acted correctly on the power flow to improve the voltage profile of the test system, without the help of the PSS and POD controllers, the GUPFC is not efficient to insert damping and stabilize the system, and other control measures are necessary to ensure adequate damping rates to the system.

Note that three local modes are unstable $\left(L_{5}, L_{6}\right.$, and $\left.L_{7}\right)$ as they have real positive parts, thereby characterizing instability in the EPS. In addition, the interarea mode $I_{9}$ is weakly damped. To solve this problem, eight PSSs were installed in the control loops of the AVRs of generating units $\mathrm{G} 1, \mathrm{G} 2, \mathrm{G} 3, \mathrm{G} 4, \mathrm{G} 5, \mathrm{G} 7, \mathrm{G} 8$, and G9; a POD controller was installed in the GUPFC control loop. The participation factors determined the locations of the PSSs in the electrical system, which indicated the generators that were more involved in the formation of each local mode. The sections that follow will discuss the tuning of these controllers.

1) Performance Evaluation of the Optimization Methods: To evaluate the performance of the algorithms, the following was considered: 1) 100 tests limited to a maximum of 1000 evaluations of the objective function given by (15) and subject to the restrictions given in (16)-(19) were carried out; and 2) two desired damping factors were considered $\left(\xi^{\text {des }} \geq 10 \%\right.$ and $\xi^{\text {des }} \geq 15 \%$ ) for all poles of interest in order to require a greater effort of the analyzed algorithms. The desired damping values $\left(\xi^{\text {des }}\right)$ were chosen based in some works with characteristics similar to those presented [13] and [18].

All the algorithms used an initial population of 20 individuals.

The PSO considered in this study is based on the standard version of this algorithm, with acceleration constants $\left(\rho_{1}=\rho_{2}=2.05\right)$, and the inertia weight decreasing linearly with the number of iterations $(\delta \in[0.5,1.5])[13]$. 


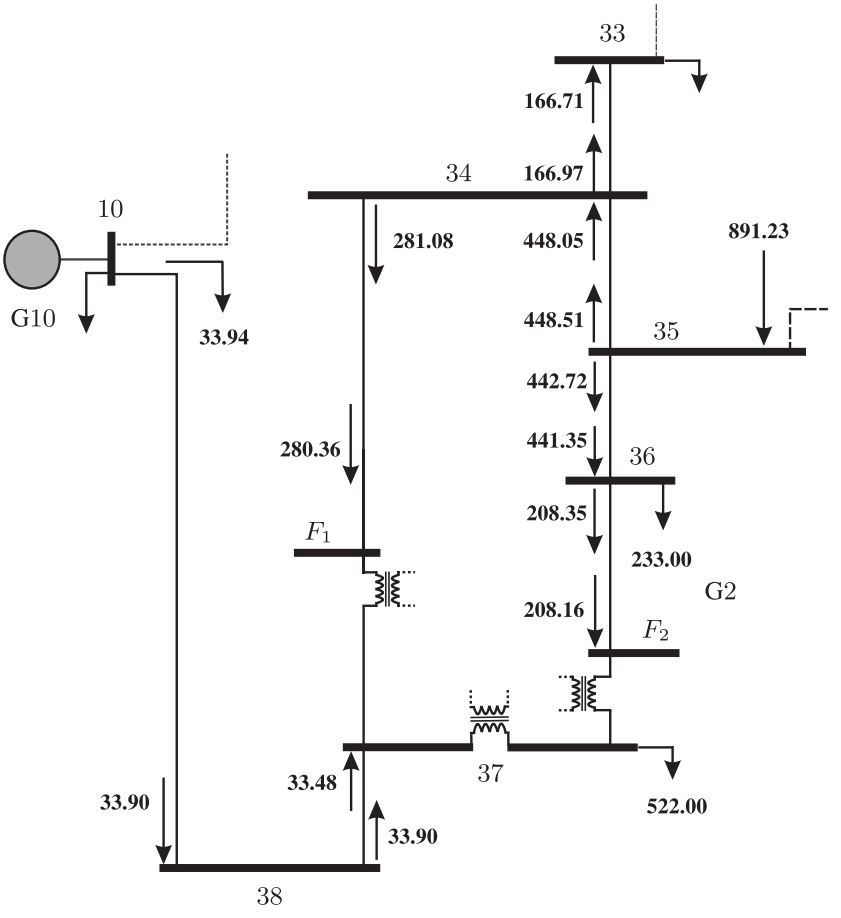

(a)

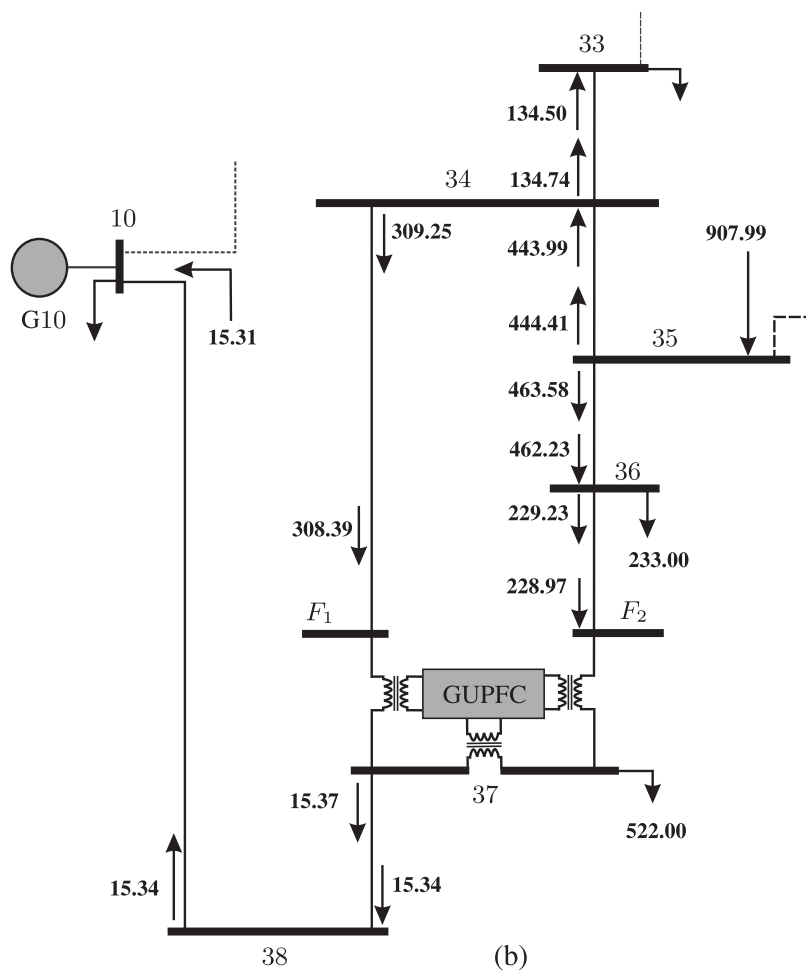

(b)

Fig. 8. Active power flow control performed by the GUPFC in the New England system. System (a) without the GUPFC and (b) with the GUPFC.

TABLE II

LiMITS OF THE PARAMETERS OF THE PSS AND POD CONTROLLERS

\begin{tabular}{ccccccc}
\hline Limit & $T_{1 n}$ & $T_{2 n}$ & $T_{p 1}$ & $T_{p 2}$ & $K_{p s s}$ & $K_{p o d}$ \\
\hline Lower & 0.10 & 0.01 & 0.05 & 0.10 & 1.00 & 0.05 \\
Upper & 1.50 & 0.10 & 0.10 & 1.00 & 10.0 & 0.50 \\
\hline
\end{tabular}

The BF-PSO use the same set of the PSO parameters described previously, while the parameters related to $\mathrm{BFO}$ were based on [15]: the number of chemotaxis steps $\left(N_{C}=10\right)$, the number of elimination and dispersion events $\left(N_{e d}=2\right)$, the number of reproduction steps $\left(N_{r e}=4\right)$, and the probability of elimination and dispersion $\left(p_{e d}=0.25\right)$.

The BA, which can be found in [22], employed the following initial values: loudness $A_{0}=0.25$ and pulse emission rate $r_{0}=0.5$ with the frequency varying within the following range: $f_{\text {min }}=0$ and $f_{\max }=2 \mathrm{~Hz}$; and $\mu=\gamma=0.9$.

Finally, the NBA uses the same values for the parameters common to the classic BA. The values of the additional parameters are based on [23]: $P \in[0.5,0.9], G=10, C \in[0.1,0.9], \Phi \in$ $[0.5,1]$, and $w \in[0.4,0.9]$.

Table II defines the limits of the time constants (seconds) and gains (p.u.) of the PSS and POD controllers. In addition, the parameters of the PI controllers of the dynamic model of the GUPFC (see Fig. 2) were fixed, and they can be seen in Table III.

This paper considers convergence to obtain a feasible solution that meets the objective function within the limit of 1000 evaluations. In Table IV, only the percentage of times that each
TABLE III

PARAMETERS OF THE PI CONTROLLERS

\begin{tabular}{cccccccc}
\hline$T_{1}^{p i}$ & $T_{2}^{p i}$ & $T_{3}^{p i}$ & $T_{4}^{p i}$ & $K_{1}^{p i}$ & $K_{2}^{p i}$ & $K_{3}^{p i}$ & $K_{4}^{p i}$ \\
\hline 0.1 & 0.1 & 0.1 & 0.1 & 0.5 & 0.5 & 0.5 & 0.5 \\
\hline
\end{tabular}

algorithm has achieved convergence is shown. In addition, for the number of iterations (average, maximum, median, and standard deviation) and for the convergence average time, this study only considered the tests in which the algorithms converged.

All algorithms tested (PSO, BF-PSO, BA, and NBA) achieved $100 \%$ convergence for the first damping factor, $\xi^{\text {des }} \geq 10 \%$. The PSO (57.77s) and the BF-PSO (38.21s) showed average times of convergence higher that of BA (13.61s) and NBA (12.71s). With an average time of 12.71 s (see Table IV), it is evident that the NBA is able to find a feasible fit for the supplementary controllers in less time, thereby decreasing the computational effort. Another difference between the algorithms tested is related to the number of iterations necessary to achieve convergence. For both damping ranges ( $\xi^{\text {des }} \geq 10 \%$ and $\xi^{\text {des }} \geq 15 \%$ ), when the average number of iterations is compared, the NBA algorithm performance is better than the other algorithms. The medians and the standard deviations (Std.) also corroborate the previous statement.

The second damping factor $\xi^{\text {des }} \geq 15 \%$ shows a large reduction in the convergence of the tested algorithms (PSO$42 \%$, BF-PSO-70\%, BA-75\%). The exception was the NBA, which maintained $100 \%$ of convergence and a low average time (18.27 s) to find a feasible solution. The average number of 
TABLE IV

COMPARISON OF THE PERFORMANCE OF ALGORITHMS

\begin{tabular}{|c|c|c|c|c|c|c|c|}
\hline \multirow[t]{2}{*}{ Method } & \multirow[t]{2}{*}{$\xi^{\text {des }}$} & \multirow[t]{2}{*}{ Converged tests $(\%)$} & \multicolumn{4}{|c|}{ Number of Iterations } & \multirow[t]{2}{*}{ Avg. Time (s) } \\
\hline & & & Avg. & Max. & Median & Std. & \\
\hline \multirow{2}{*}{ PSO } & $\geq 10 \%$ & 100 & 160 & 631 & 116 & 105 & 57.8 \\
\hline & $\geq 15 \%$ & 42 & 419 & 979 & 376 & 237 & 180.5 \\
\hline \multirow{2}{*}{ BF-PSO } & $>10 \%$ & 100 & 93 & 878 & 26 & 102 & 38.2 \\
\hline & $\geq 15 \%$ & 70 & 342 & 987 & 226 & 239 & 137.1 \\
\hline \multirow{2}{*}{$\mathrm{BA}$} & $\geq 10 \%$ & 100 & 35 & 67 & 31 & 6 & 13.6 \\
\hline & $\geq 15 \%$ & 75 & 52 & 142 & 42 & 21 & 21.2 \\
\hline \multirow{2}{*}{ NBA } & $\geq 10 \%$ & 100 & 33 & 44 & 32 & 2 & 12.7 \\
\hline & $\geq 15 \%$ & 100 & 42 & 144 & 37 & 11 & 18.3 \\
\hline
\end{tabular}

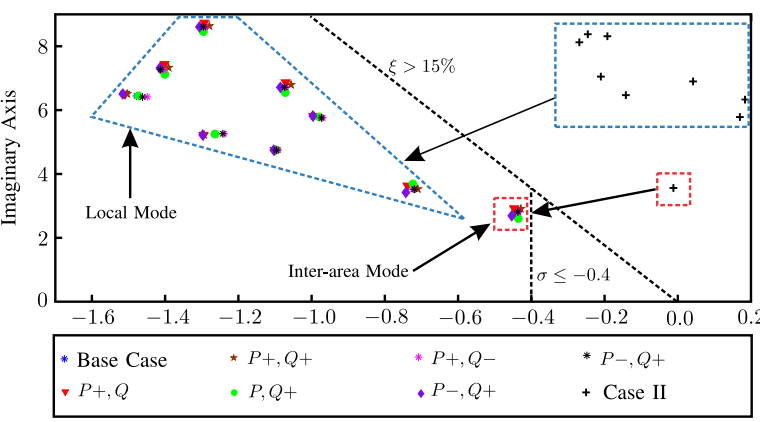

(a)

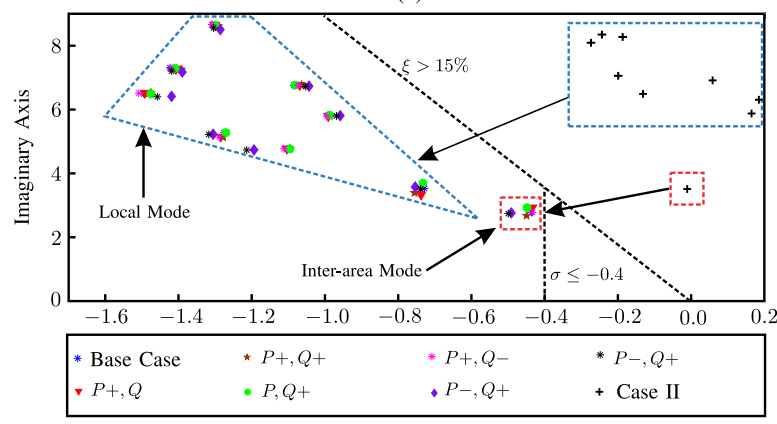

(c)

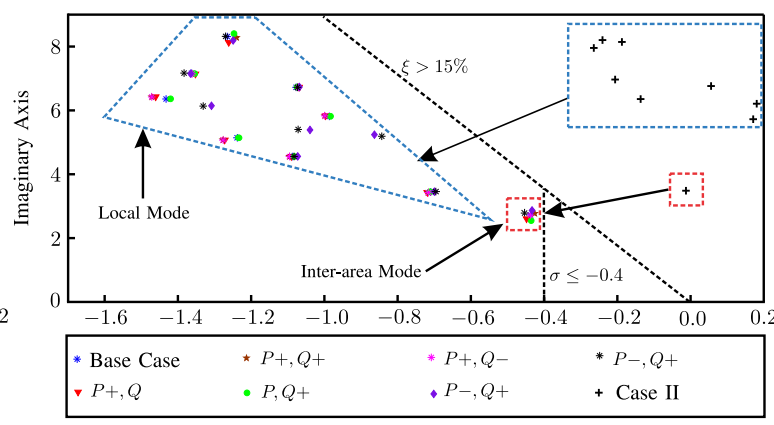

(b)

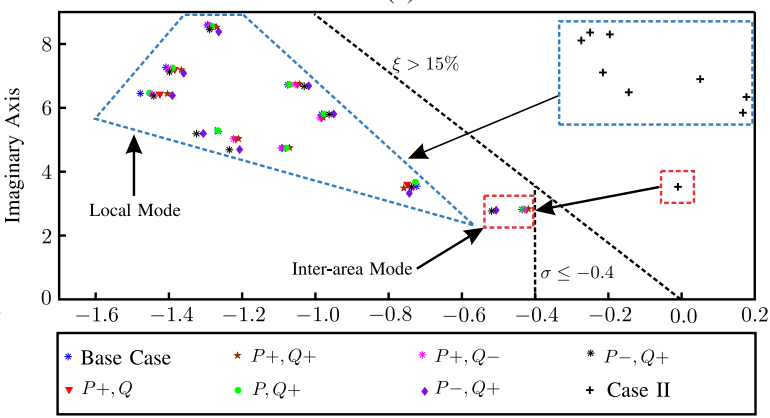

(d)

Fig. 9. Region of pole allocation. (a) Variation in load buses of 2.5\%. (b) Variation in load buses of 5.0\%. (c) Variation in load buses of 7.5\%. (d) Variation in load buses of $10.0 \%$.

TABLE V

PARAMETERS OF THE PSS AND GUPFC-POD CONTROLLERS

\begin{tabular}{llll}
\hline Device & $T_{1}=T_{3}$ & $T_{2}=T_{4}$ & $K$ \\
\hline PSS G1 & 1.1116 & 0.0592 & 6.953 \\
PSS G2 & 0.7190 & 0.0490 & 6.541 \\
PSS G3 & 0.6853 & 0.0338 & 8.593 \\
PSS G4 & 0.6302 & 0.0345 & 6.078 \\
PSS G5 & 0.5500 & 0.0412 & 9.900 \\
PSS G7 & 0.5174 & 0.0503 & 8.747 \\
PSS G8 & 0.6570 & 0.0365 & 9.229 \\
PSS G9 & 0.3919 & 0.0882 & 6.184 \\
GUPFC-POD & 0.1735 & 0.2382 & 0.225 \\
\hline
\end{tabular}

TABLE VI

DOMINANT EIGENVALUES, DAMPING COEFFICIENTS $(\xi)$, AND NATURAL UNDAMPED FREQUENCIES $\left(\omega_{n}\right)$ CONSIDERING THE EFFECT OF THE CONTROLlERS

\begin{tabular}{llll}
\hline Modes & Eigenvalues & $\xi$ (p.u.) & $\omega_{n}(\mathrm{~Hz})$ \\
\hline$L_{1}$ & $-1.289 \pm j 8.475$ & 0.1504 & 1.364 \\
$L_{2}$ & $-1.393 \pm j 7.259$ & 0.1885 & 1.176 \\
$L_{3}$ & $-1.089 \pm j 6.761$ & 0.1590 & 1.089 \\
$L_{4}$ & $-1.457 \pm j 6.516$ & 0.2182 & 1.063 \\
$L_{5}$ & $-1.004 \pm j 5.916$ & 0.1674 & 0.955 \\
$L_{6}$ & $-1.266 \pm j 5.246$ & 0.2354 & 0.859 \\
$L_{7}$ & $-1.098 \pm j 4.748$ & 0.2253 & 0.776 \\
$L_{8}$ & $-0.965 \pm j 5.028$ & 0.1884 & 0.815 \\
$I_{9}$ & $-0.723 \pm j 3.521$ & 0.2011 & 0.572 \\
\hline
\end{tabular}




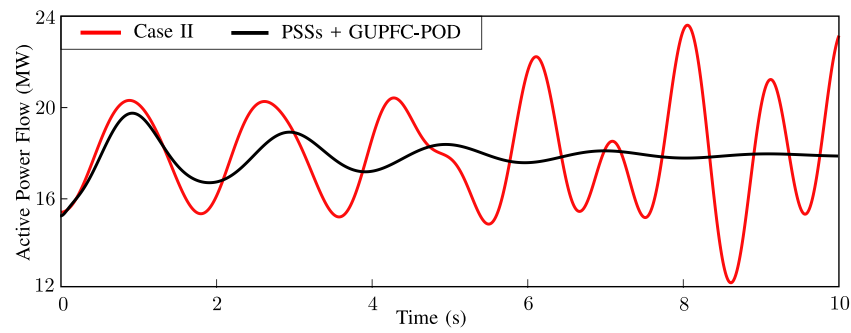

Fig. 10. Active power flow between buses $37-38$.
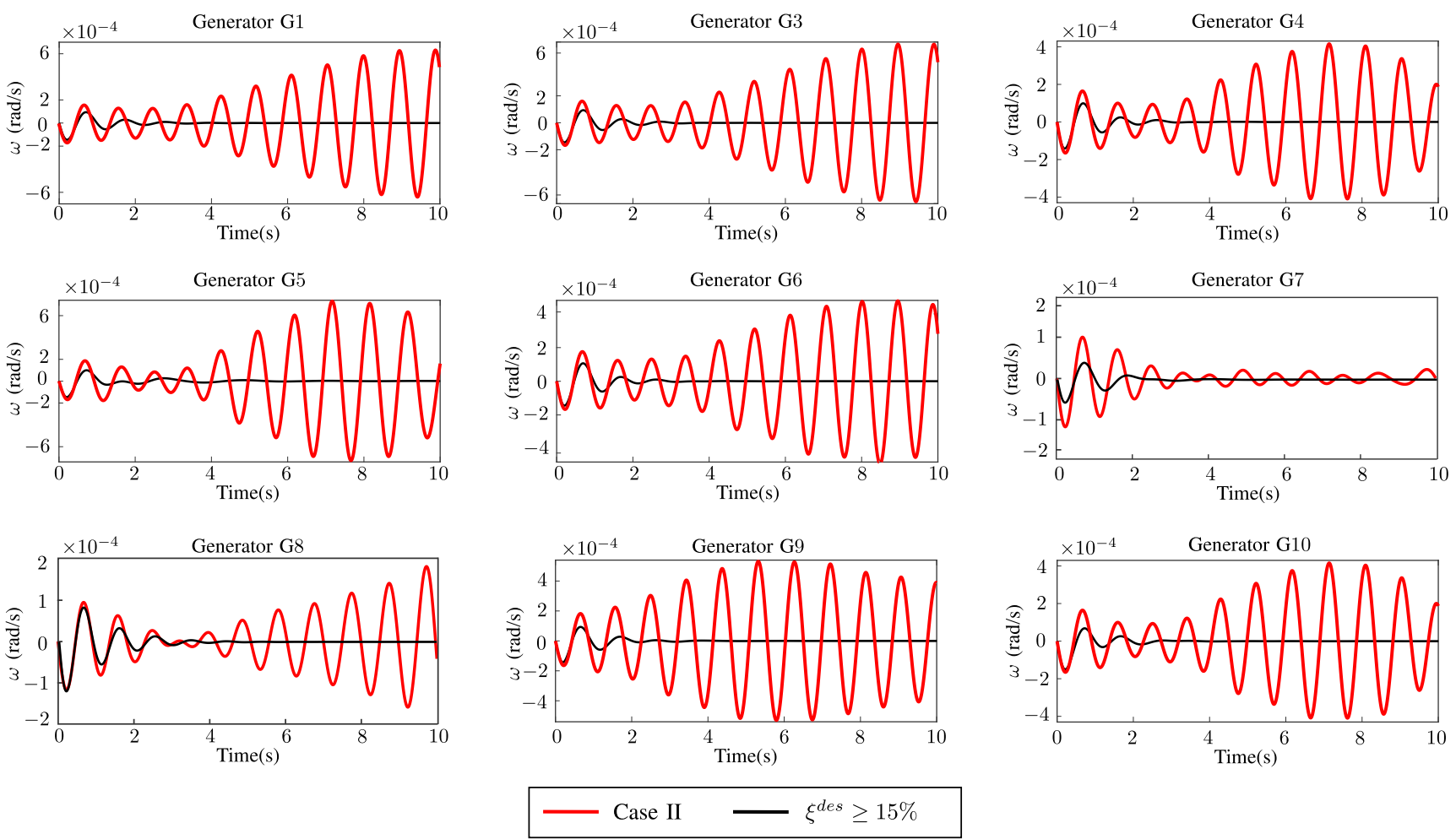

Fig. 11. Variation in the angular speeds of the rotors of the SMs of the test system.

iterations increased more than twice for PSO and more than three times for BF-PSO compared to the first damping factor. It is observed that for the more rigorous damping factor ( $\xi^{\text {des }} \geq 15 \%$ ), the improvements implemented in the NBA in relation to habitat diversification, local search intensification, and adaptive compensation of the Doppler effect in echo contribute to the better performance of this technique with respect to the other techniques evaluated, as the statistical metrics presented in Table IV show.

2) Evaluation of the Proposed Technique for Different Loading Scenarios: Table V shows a setting, randomly selected among the 100 valid tests found by the NBA during the simulations to tune the PSSs and GUPFC-POD controllers, i.e., the fit found satisfies (15) and the bounds shown in Tables II and III. By setting the New England system with these parameters, new dominant eigenvalues, damping coefficients, and natural undamped frequencies were calculated, obtained through simulations and shown in Table VI with one new operational status, with at least $15 \%$ of damping to all local and interarea oscillation modes.

To prove the robustness of the solutions found by the NBA, the operating point of the test system varied to create different load scenarios. A setting, randomly selected from the 100 feasible tests, was used to tune the PSSs and POD controllers (see Table V). In addition to the base case and the Case II, 24 additional load scenarios were considered by utilizing four different increment factors for active and reactive loads $[2.5 \%, 5.0 \%, 7.5 \%$, and $10.0 \%$ ] and a set of six different combinations of active and reactive power variations in all load buses $[(P+, Q)$, $(P+, Q+),(P, Q+),(P+, Q-),(P-, Q+),(P-, Q-)]$.

Fig. 9 reveals the allocation of the eigenvalues of interest within a region determined by the constraints discussed in Section IV. For all scenarios, the eigenvalues of interest were shifted to the left half-plane of the complex plane, according to the desired damping and the constraint that determines a minimum value for the real part of the eigenvalues $\left(\sigma_{i} \leq-0.4\right)$. By an- 
alyzing Fig. 9, it can be verified that the damping levels in the New England system remained almost unchanged, even after the variations of the loads. In other words, the New England system was still able to operate with high damping levels for different load scenarios, evidencing the robustness of the parameters provided by the NBA.

In order to analyze the stability in the time domain, a perturbation (step of 0.05 p.u.) was applied to the mechanical power of generator G2 (reference generator). This perturbation can be compared with a small variation in the system load, which will result in an adjustment of the generation. A small variation in the system load can be considered an event common throughout the day. For the next simulations were considered two cases: Case II (without supplementary damping controllers) and, the case with controllers acting in the test system previously tuned by NBA (see Table V) to provide a desired minimum damping ( $\xi^{\text {des }} \geq$ $15 \%$ ). Fig. 10 shows the active power flow between buses 37 and 38 after a perturbation simulating an abrupt variation of the system load. Fig. 8(b) shows the active power flow (15.37 MW) controlled by the GUPFC between bars 37 and 38 before disturbance. From the analysis of Fig. 10, it is observed that after the disturbance, the active power flow stabilizes quickly at a new equilibrium point (approximately $18 \mathrm{MW}$ ) for the case where the PSSs and GUPFC-POD controllers are acting on the power system, whereas for Case II the system becomes unstable.

In the same context, Fig. 11 shows the variation of the angular velocity of the rotors of all synchronous machines (SMs) of the New England system in relation to the variation of the rotor angular velocity of the reference generator after the perturbation. The curve in red represent the Case II, while the black curve represent the case in which the system operates with high stability margin, equipped with eight PSS controllers and one GUPFCPOD set, with parameters designed using the NBA. Note that after the perturbation, the curves representing the Case II show an unstable system or with low damped, while the second case, even after the perturbation, the analysis of the obtained curves shows that the system is well damped, evidencing its high margin of stability to small-signal perturbations.

\section{CONCLUSION}

This paper proposed the coordinated tuning of the parameters of the PSS and GUPFC-POD supplementary damping controllers using an NBA-based technique. The objective was to insert additional damping to low-frequency electromechanical oscillations, local and interarea modes, present in the multimachine power system.

The NBA performance was compared with three other natureinspired optimization methods: PSO, BF-PSO, and BA. The analysis of the statistical results showed a better NBA performance, with high convergence rates and a smaller number of iterations to achieve the desired damping for the system. In large part, this is due to the modifications implemented in the NBA to avoid the entrapment of the bat (solution of the problem) into a local optimal point. In order to evaluate the robustness of the solutions found, different load scenarios were considered, and for all scenarios, stability was maintained with damping levels above those specified by the design. In order to analyze the stability in the time domain, simulations were performed to evaluate the behavior of the system after a disturbance. Again, the results demonstrated the efficiency of the proposed optimization method for tuning the supplementary damping controllers.

Finally, the performance of GUPFC installed in the New England system to improve the overall voltage profile, and their influence on improving the damping of the oscillatory modes was also evaluated. It can be concluded that the device was effective in overall improvement of voltage levels in all buses of the test system, after power flow control. However, it was observed that the GUPFC acting alone (without the PSS and POD controllers) did not provide sufficient damping to low frequency oscillations present in the New England system.

\section{REFERENCES}

[1] P. Kundur et al., "Definition and classification of power system stability IEEE/CIGRE joint task force on stability terms and definitions," IEEE Trans. Power Syst., vol. 19, no. 3, pp. 1387-1401, Aug. 2004.

[2] S. M. Deckmann and V. F. Da Costa, "A power sensitivity model for electromechanical oscillation studies," IEEE Trans. Power Syst., vol. 9, no. 2, pp. 965-971, May 1994.

[3] P. Kundur, M. Klein, G. J. Rogers, and M. S. Zywno, "Application of power system stabilizers for enhancement of overall system stability," IEEE Trans. Power Syst., vol. 4, no. 2, pp. 614-626, May 1989.

[4] F. P. De Mello and C. Concordia, "Concepts of synchronous machine stability as affected by excitation control," IEEE Trans. Power App. Syst., vol. 88, no. 4, pp. 316-329, Apr. 1969.

[5] E. Larsen and D. Swann, "Applying power system stabilizers part II: Performance objectives and tuning concepts," IEEE Trans. Power App. Syst., vol. PAS-100, no. 6, pp. 3025-3033, Jun. 1981.

[6] R. Yousefian, A. Sahami, and S. Kamalasadan, "Hybrid transient energy function-based real-time optimal wide-area damping controller," IEEE Trans. Ind. Appl., vol. 53, no. 2, pp. 1506-1516, Mar./Apr. 2017.

[7] T. Surinkaew and I. Ngamroo, "Hierarchical co-ordinated wide area and local controls of DFIG wind turbine and PSS for robust power oscillation damping," IEEE Trans. Sustain. Energy, vol. 7, no. 3, pp. 943-955, Jul. 2016.

[8] N. Kulkarni, S. Kamalasadan, and S. Ghosh, "An integrated method for optimal placement and tuning of a power system stabilizer based on full controllability index and generator participation," IEEE Trans. Ind. Appl., vol. 51, no. 5, pp. 4201-4211, Sep./Oct. 2015.

[9] S.-M. Baek, J.-W. Park, and I. A. Hiskens, "Optimal tuning for linear and nonlinear parameters of power system stabilizers in hybrid system modeling," IEEE Trans. Ind. Appl., vol. 45, no. 1, pp. 87-97, Jan./Feb. 2009.

[10] N. G. Hingorani and L. Gyugyi, Understanding FACTS: Concepts and Technology of Flexible AC Transmission System. Hoboken, NJ, USA: IEEE, 2000.

[11] M. Noroozian and G. Andersson, "Damping of power system oscillations by use of controllable components," IEEE Trans. Power Del., vol. 9, no. 4, pp. 2046-2054, Oct. 1994

[12] L. H. Hassan, M. Moghavvemi, H. A. F. Almurib, and K. M. Muttaqi, "A coordinated design of PSSs and UPFC-based stabilizer using genetic algorithm," IEEE Trans. Ind. Appl., vol. 50, no. 5, pp. 2957-2966, Sep. 2014.

[13] E. V. Fortes, P. B. Araujo, and L. H. Macedo, "Coordinated tuning of the parameters of PI, PSS and POD controllers using a specialized ChuBeasley's genetic algorithm," Elect. Power Syst. Res., vol. 140, pp. 708$721,2016$.

[14] L. H. Hassan, M. Moghavvemi, H. A. Almurib, K. Muttaqi, and V. G. Ganapathy, "Optimization of power system stabilizers using participation factor and genetic algorithm," Int. J. Elect. Power Energy Syst., vol. 55, pp. 668-679, 2014.

[15] E. Ali and S. Abd-Elazim, "TCSC damping controller design based on bacteria foraging optimization algorithm for a multimachine power system," Int. J. Elect. Power Energy Syst., vol. 37, no. 1, pp. 23-30, 2012 . 
[16] E. L. Miotto, P. B. de Araujo, B. R. Gamino, E. V. Fortes, and L. F. B. Martins, "Coordinated tuning of the parameters of supplementary controllers damping using bio-inspired algorithms," in Proc. 2016 12th IEEE Int. Conf. Ind. Appl., 2016, pp. 1-8.

[17] H. Hasanvand, M. R. Arvan, B. Mozafari, and T. Amraee, "Coordinated design of PSS and TCSC to mitigate interarea oscillations," Int. J. Elect. Power Energy Syst., vol. 78, pp. 194-206, 2016.

[18] L. F. B. Martins, P. B. de Araujo, E. V. de Fortes, and L. H. Macedo, "Design of the PI-UPFC-POD and PSS damping controllers using an artificial bee colony algorithm," J. Control, Autom. Elect. Syst., vol. 28, pp. 762-773, Sep. 2017.

[19] S. A. Elazim and E. Ali, "Optimal power system stabilizers design via cuckoo search algorithm," Int. J. Elect. Power Energy Syst., vol. 75, pp. 99 107, 2016.

[20] S. Panda, N. K. Yegireddy, and S. K. Mohapatra, "Hybrid BFOA-PSO approach for coordinated design of PSS and SSSC-based controller considering time delays," Int. J. Elect. Power Energy Syst., vol. 49, pp. 221-233, 2013.

[21] S. Abd-Elazim and E. Ali, "Synergy of particle swarm optimization and bacterial foraging for TCSC damping controller design," Int. J. WSEAS Trans. Power Syst., vol. 8, no. 2, pp. 74-84, 2013.

[22] W. Peres, E. J. de Oliveira, J. A. P. Filho, and I. C. da Silva Junior, "Coordinated tuning of power system stabilizers using bio-inspired algorithms," Int. J. Elect. Power Energy Syst., vol. 64, pp. 419-428, 2015.

[23] X.-B. Meng, X. Z. Gao, Y. Liu, and H. Zhang, "A novel bat algorithm with habitat selection and doppler effect in echoes for optimization," Expert Syst. Appl., vol. 42, no. 17-18, pp. 6350-6364, 2015.

[24] R. S. Lubis, S. P. Hadi, and Tumiran,"Modeling of the generalized unified power flow controller for optimal power flow," in Proc. 2011 Int. Conf. Elect. Eng. Informat., Jul. 2011, pp. 1-6.

[25] P. Kundur, Power System Stability and Control. New York, NY, USA: MacGraw-Hill, 1994.

[26] J. Kennedy and R. Eberhart, "Particle swarm optimization," in Proc. IEEE Int. Conf. Neural Netw., vol. 4, 1995, pp. 1942-1948.

[27] D. Bratton and J. Kennedy, "Defining a standard for particle swarm optimization," in Proc. Swarm Intell. Symp., 2007, pp. 120-127.

[28] K. Passino, "Biomimicry of bacterial foraging for distributed optimization and control," IEEE Control Syst., vol. 22, no. 3, pp. 52-67, Jun. 2002.

[29] X.-S. Yang, Nature-Inspired Metaheuristic Algorithms, 2nd ed. University of Cambrige, Cambridge, U.K.: Luniver, 2010.

[30] J. Sun, W. Fang, X. Wu, V. Palade, and W. Xu, "Quantum-behaved particle swarm optimization: Analysis of individual particle behavior and parameter selection," Evol. Comput., vol. 20, no. 3, pp. 349-393, 2012.

[31] C. Canizares et al., "Benchmark models for the analysis and control of small-signal oscillatory dynamics in power systems," IEEE Trans. Power Syst., vol. 32, no. 1, pp. 715-722, Jan. 2017.

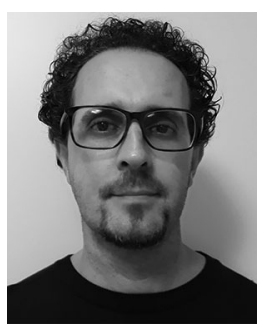

Ednei Luiz Miotto received the B.S. degree in electrical engineering from the State University of Western Paraná, Foz do Iguaçu, Brazil, in 2003, and the M.S. degree in electrical engineering from the State University of Londrina, Londrina, Brazil, in 2010. $\mathrm{He}$ is currently working toward the Ph.D. degree in electrical engineering with São Paulo State University, Ilha Solteira, Brazil.

$\mathrm{He}$ is a Professor with the Paraná Federal University of Technology, Paraná, Brazil. His current research interests include small-signal stability analysis in power systems.

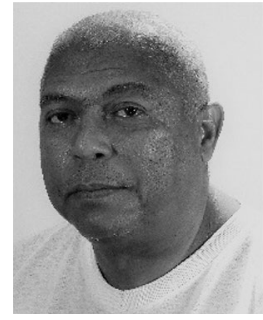

Percival Bueno de Araujo received the B.S. degree from the São Paulo State University Júlio de Mesquita Filho, in 1982, the M.S. degree from Santa Catarina Federal University, in 1988, the Ph.D. degree from São Paulo University, in 1998, and the Ph.D. degree from the Universidad de Castilla - La Mancha, in 2013, all in electrical engineering.

He is currently an Assistant Professor with São Paulo State University Júlio de Mesquita Filho. His research interests include electric power systems, power systems stabilizers, and dynamic stability.

Dr. Bueno de Araujo is a Reviewer for the IEEE TRANSACTIONS ON POWER SYSTEMS, the International Journal of Electrical Power and Energy Systems, IEEE Latin America, among others.

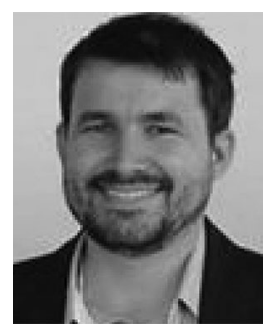

Elenilson de Vargas Fortes received the Licenciate degree in mathematics from the Federal University of Espírito Santo, in 2004, the M.S. degree in mathematics from the Federal University of Braslia, in 2007, and the Ph.D. degree in electrical engineering from São Paulo State University, Ilha Solteira, Brazil, in 2016.

He is currently a Professor with the Goiás Federal Institute of Education, Science, and Technology. His current research interests include small-signal stability analysis in power systems.

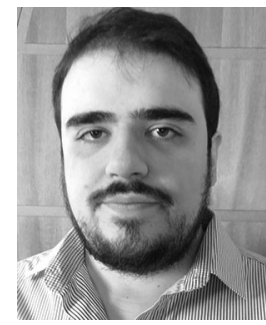

Bruno Rafael Gamino received the B.S. degree in electrical engineering from São Paulo State University, Ilha Solteira, Brazil, in 2014, where he is working toward the Ph.D. degree.

He is currently a Professor with the Federal Institute of São Paulo, Birigui, Brazil. His research interests include electric power systems, small-signal stability, metaheuristics, and optimization methods.

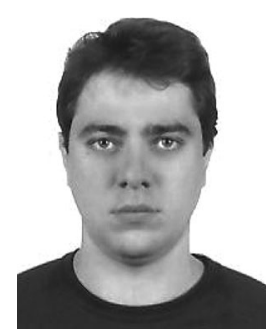

Luís Fabiano Barone Martins received the B.S. and Ph.D. degrees in electrical engineering from São Paulo State University, Ilha Solteira, Brazil, in 2004 and 2017, respectively, and the M.S. degree in electrical engineering from São Paulo State University, Bauru, Brazil, in 2011.

He is a Professor with the Paraná Federal Institute of Education, Science, and Technology. His current research interests include small-signal stability analysis in power systems. 